ESAIM: M2AN

Vol. 40, No 6, 2006, pp. 1053-1067

DOI: $10.1051 / \mathrm{m} 2 \mathrm{an}: 2007002$
ESAIM: Mathematical Modelling and Numerical Analysis

www.edpsciences.org/m2an

\title{
A SECOND-ORDER FINITE VOLUME ELEMENT METHOD ON QUADRILATERAL MESHES FOR ELLIPTIC EQUATIONS
}

\author{
MiN YANG ${ }^{1}$
}

\begin{abstract}
In this paper, by use of affine biquadratic elements, we construct and analyze a finite volume element scheme for elliptic equations on quadrilateral meshes. The scheme is shown to be of second-order in $H^{1}$-norm, provided that each quadrilateral in partition is almost a parallelogram. Numerical experiments are presented to confirm the usefulness and efficiency of the method.
\end{abstract}

Mathematics Subject Classification. 65N30, 65N15.

Received: April 26, 2006. Revised: August 7, and October 21, 2006.

\section{INTRODUCTION}

Consider the following elliptic boundary value problem:

$$
\begin{aligned}
-\nabla \cdot(p(X) \nabla u) & =f(X), & & X \in \Omega, \\
u(X) & =0, & & X \in \partial \Omega,
\end{aligned}
$$

where $\Omega$ is a bounded polygonal domain in $\mathrm{R}^{2}$ with boundary $\partial \Omega$ and $X=(x, y)$. Assume that $f(X) \in L^{2}(\Omega)$, $p(X)$ is Lipschitz continuous with the Lipschitz constant $L$, and $0<p_{*} \leq p(X) \leq p^{*}$. Here $p_{*}$ and $p^{*}$ are positive constants.

Finite volume element (FVE) methods $[3,6,7,16,17]$, also named as generalized difference methods $[13,14,19$, $22]$ or box methods [1,11], have been widely used in several engineering fields, such as fluid mechanics, heat and mass transfer or petroleum engineering. The FVE methods involve two spaces. One is the solution space $S_{h}$ of piecewise polynomial functions over the primal partition, and another is the test space $S_{h}^{*}$ of piecewise constant functions over the dual partition. Similar as the finite element methods the unknowns are approximated by a Galerkin expansion. FVE procedures are usually easier to implement than finite element procedures and offer most of the advantages of flexibility for handling complicated domain geometries. More important, the test space $S_{h}^{*}$ ensures the local conservation property of the methods, which is highly desirable in computational conservation laws.

But an undesirable property of the FVE methods is that multidimensional FVE schemes are usually only first-order accurate. For some details we refer to the articles on triangular meshes $[3,16]$ or on quadrilateral meshes $[6,13]$. In present time, one approach to obtain higher order schemes is using uniform or symmetric meshes to obtain superconvergence. The FVE approximations for elliptic and integro differential equations on

Keywords and phrases. Finite volume element, second-order, quadrilateral meshes, error estimates.

1 Department of Mathematics, Yantai University, Yantai, 264005, P.R. China. yangmin_math@163.com

(c) EDP Sciences, SMAI 2007 
such meshes have been considered in $[4,7,9,10]$. However, for complex geometries, it seems difficult to generate these meshes. Another approach is choosing higher order finite elements as solution space. This approach seems more suitable to handle complex geometries. FVE discretization for Poisson equations, with quadratic basis on different dual partitions, was developed in $[15,19]$. Then the method was extended to analyze some nonlinear convection diffusion problems in [23]. In [20,21], such approach is adopted to construct more general higher order schemes on unstructured grids, but no theoretical analysis was presented. By use of Gauss integration and approximation replacement of the weak forms, analysis of a discrete biquadratic FVE scheme on rectangular meshes was presented for elliptic problems in [22]. In this paper, we will put forward a second order FVE method for elliptic problems on more general quadrilateral meshes. By use of the properties of affine mapping, we obtain the optimal error estimates in $H^{1}$ norm of the scheme under some "almost parallelogram" assumptions about the meshes.

The rest of the paper is organized as follows. In the next section we introduce some necessary notations and obtain a discrete FVE scheme for problem (1). In Section 3, some auxiliary results about the grids and the FVE weak form are proved. Then we prove an optimal $H^{1}$-error estimates of second-order under some regularity condition. In the last section, numerical examples are presented on uniform and nonuniform quadrilateral meshes to show the efficiency of the method.

Throughout this paper we use $C$ to denote a generic positive constant independent of discretization parameters.

\section{Meshes AND NOTATION}

Let $T_{h}=\{Q\}$ be a quadrilateral partition of $\Omega$, which is named primal partition, where the intersection of any two closed quadrilaterals is either an entire side or a common vertex. Let $h_{Q}$ denote the diameter of the element $Q$ and $\rho_{Q}$ denote the maximum diameter of circles contained in $Q$. We denote by $m(Q)$ the measure of $Q$.

The primal partition $T_{h}$ is assumed to be regular, i.e., there exists a positive constant $C^{*}$ such that

$$
\frac{h_{Q}}{\rho_{Q}} \leq C^{*}, \quad \forall Q \in T_{h}
$$

We also assume that each quadrilateral $Q$ in $T_{h}$ is close to a parallelogram, i.e., (see Fig. 1)

$$
\left|\overrightarrow{P_{1} P_{4}}+\overrightarrow{P_{3} P_{2}}\right|=O\left(h^{2}\right)
$$

where $h=\max \left\{h_{Q} \mid Q \in T_{h}\right\}$.

Remark 2.1. We can obtain a family of almost parallelograms satisfying the condition (3) by recursively refining each quadrilateral in any reasonable coarser grid into four smaller quadrilaterals by connecting the opposite midpoints.

Let $\widehat{Q}=[0,1] \times[0,1]$ in the $\hat{x} \hat{y}$-plane be a reference element with the vertices

$$
\hat{P}_{1}=(0,0), \quad \hat{P}_{2}=(1,0), \quad \hat{P}_{3}=(1,1), \quad \hat{P}_{4}=(0,1) .
$$

For any quadrilateral $Q \in T_{h}$, where $P_{i}=\left(x_{i}, y_{i}\right)$, there exists a unique invertible bilinear transformation $F_{Q}$ which maps $\widehat{Q}$ onto $Q$ such that

$$
F_{Q}:\left\{\begin{array}{l}
x=x_{1}+\left(x_{2}-x_{1}\right) \hat{x}+\left(x_{4}-x_{1}\right) \hat{y}+\left(x_{1}-x_{2}+x_{3}-x_{4}\right) \hat{x} \hat{y} \\
y=y_{1}+\left(y_{2}-y_{1}\right) \hat{x}+\left(y_{4}-y_{1}\right) \hat{y}+\left(y_{1}-y_{2}+y_{3}-y_{4}\right) \hat{x} \hat{y}
\end{array}\right.
$$

Let $\mathcal{J}_{F_{Q}}$ denote the Jacobian matrix of $F_{Q}$ at $\hat{X}$ and $J_{F_{Q}}=\operatorname{det} \mathcal{J}_{F_{Q}}$. Let $\mathcal{J}_{F_{Q}^{-1}}$ denote the Jacobian matrix of $F_{Q}^{-1}$ at $X$ and $J_{F_{Q}^{-1}}=\operatorname{det} \mathcal{J}_{F_{Q}^{-1}}$. 


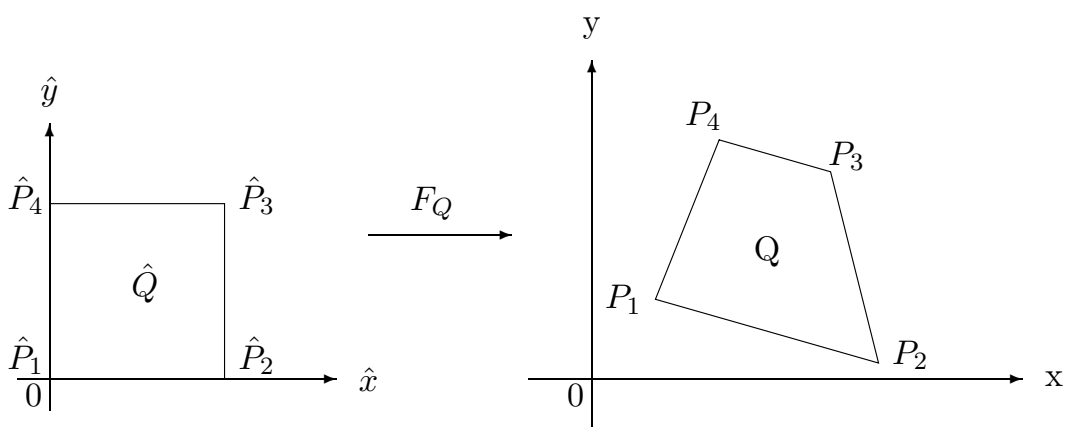

Figure 1. The bilinear mapping $F_{Q}: \widehat{Q} \rightarrow Q$.

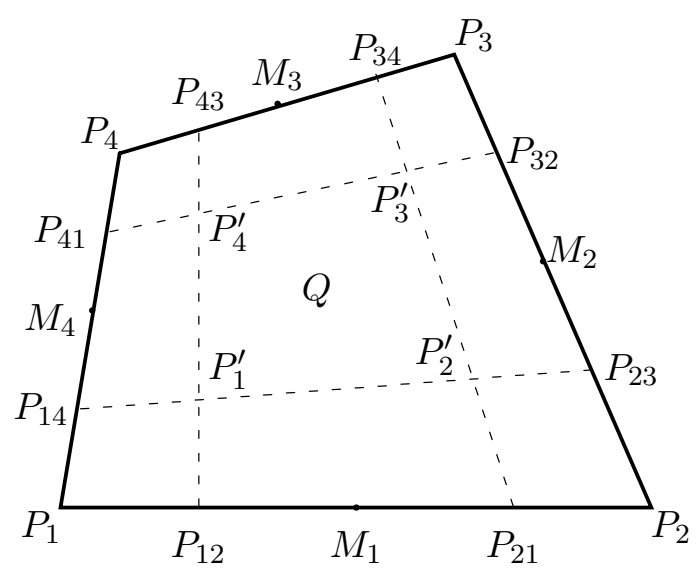

Figure 2. Control volumes in a quadrilateral element.

The approximate solution of (1) will be sought in the discrete space $S_{h}$ defined below. Let $S_{h}(\widehat{Q})$ be the standard biquadratic polynomial space on the reference element $\widehat{Q}$. Then set

$$
S_{h}=\left\{u_{h} \in H_{0}^{1}(\Omega):\left.u_{h}\right|_{Q}=\hat{u}_{h} \circ F_{Q}^{-1}, \hat{u}_{h} \in S_{h}(\widehat{Q}), Q \in T_{h}\right\}
$$

In order to establish the FVE scheme, we shall introduce a dual partition $T_{h}^{*}$ of $T_{h}$, whose elements are called control volumes. For any element $Q \in T_{h}$ (see Fig. 2) with vertices $P_{i}, 1 \leq i \leq 4$, let $M_{i}$ denote the midpoints of four edges and $O$ denote the averaging center. On each edge, a point $P_{i j}$ is chosen such that

$$
\left|P_{i} P_{i j}\right|=\frac{1}{4}\left|P_{i} P_{j}\right|
$$

Let $i+1=1$ if $i=4$ and let $i-1=4$ if $i=1$. Then the control volume in $Q$ of a vertex $P_{i}$ is the subregion $P_{i} P_{i(i+1)} P_{i}^{\prime} P_{i(i-1)}$. The control volume in $Q$ of a midpoint $M_{i}$ is the subregion $P_{i(i+1)} P_{(i+1) i} P_{i+1}^{\prime} P_{i}^{\prime}$.

Then for each vertex $P$, we associate a control volume $Q_{P}^{*}$, which is built by the union of the above subregions, sharing the vertex $P$. For each midpoint $M$ on an element edge, we associate a control volume $Q_{M}^{*}$, which is built by the union of the above subregions, sharing a common edge including $M$. For each averaging center $O$, we associate a control volume $Q_{O}^{*}$, which is built by the subregion $P_{1}^{\prime} P_{2}^{\prime} P_{3}^{\prime} P_{4}^{\prime}$ (see Fig. 2). The collection of these control volumes makes the dual partition $T_{h}^{*}$. 
Now for any control volume $Q^{*} \in T_{h}^{*}$, integrating (1) over $Q^{*}$ and using the Green's formula, we obtain

$$
-\int_{\partial Q^{*}} p(X) \nabla u \cdot n \mathrm{~d} s=\int_{Q^{*}} f(X) \mathrm{d} X
$$

where $n$ denotes the unit outer normal vector to $\partial Q^{*}$.

Let $S_{h}^{*}$ be a piecewise constant space associated with the dual partition $T_{h}^{*}$. Multiply (5) by a test function $v \in S_{h}^{*}$ and sum the result over $T_{h}^{*}$ to yield

$$
-\sum_{Q^{*} \in T_{h}^{*}}\left(v \int_{\partial Q^{*}} p \nabla u \cdot n \mathrm{~d} s\right)=\sum_{Q^{*} \in T_{h}^{*}} v \int_{Q^{*}} f \mathrm{~d} X .
$$

Reordering by elements, we have (see Fig. 2)

$$
\begin{array}{r}
\sum_{Q^{*} \in T_{h}^{*}} v \int_{\partial Q^{*}} p \nabla u \cdot n \mathrm{~d} s=\sum_{Q \in T_{h}}\left\{\sum _ { i = 1 } ^ { 4 } \left[v\left(P_{i}\right) \int_{\partial Q_{P_{i}}^{*} \cap Q} p \nabla u \cdot n_{P_{i}} \mathrm{~d} s\right.\right. \\
\left.\left.+v\left(M_{i}\right) \int_{\partial Q_{M_{i}}^{*} \cap Q} p \nabla u \cdot n_{M_{i}} \mathrm{~d} s\right]+v(O) \int_{\partial Q_{O}^{*}} p \nabla u \cdot n_{O} \mathrm{~d} s\right\}
\end{array}
$$

where $n_{P_{i}}, n_{M_{i}}$ and $n_{O}$ denote the unit outer normal vectors of the involved integral domain.

So for $u \in H_{0}^{1}(\Omega)$ and $v \in S_{h}^{*}$, we define two bilinear forms $a_{Q}(u, v)$ and $\bar{a}_{Q}(u, v)$ as follows:

$$
\begin{aligned}
a_{Q}(u, v)= & -\sum_{i=1}^{4}\left[v\left(P_{i}\right) \int_{\partial Q_{P_{i}}^{*} \cap Q} p \nabla u \cdot n_{P_{i}} \mathrm{~d} s\right. \\
& \left.+v\left(M_{i}\right) \int_{\partial Q_{M_{i}}^{*} \cap Q} p \nabla u \cdot n_{M_{i}} \mathrm{~d} s\right]-v(O) \int_{\partial Q_{O}^{*}} p \nabla u \cdot n_{O} \mathrm{~d} s
\end{aligned}
$$

and

$$
\begin{aligned}
\bar{a}_{Q}(u, v)= & -p(O)\left\{\sum _ { i = 1 } ^ { 4 } \left[v\left(P_{i}\right) \int_{\partial Q_{P_{i}}^{*} \cap Q} \nabla u \cdot n_{P_{i}} \mathrm{~d} s\right.\right. \\
& \left.\left.+v\left(M_{i}\right) \int_{\partial Q_{M_{i}}^{*} \cap Q} \nabla u \cdot n_{M_{i}} \mathrm{~d} s\right]+v(O) \int_{\partial Q_{O}^{*}} \nabla u \cdot n_{O} \mathrm{~d} s\right\} .
\end{aligned}
$$

Let $a(u, v)=\sum_{Q \in T_{h}} a_{Q}(u, v)$ and $\bar{a}(u, v)=\sum_{Q \in T_{h}} \bar{a}_{Q}(u, v)$. We can rewrite (6) in the following FVE weak form

$$
a(u, v)=(f, v), \quad v \in S_{h}^{*}
$$

Now we introduce two interpolation operators $\Pi$ and $\Pi^{*}$, where $\Pi: H_{0}^{1}(\Omega) \rightarrow S_{h}$ satisfying [8]

$$
\|u-\Pi u\|_{H^{r}} \leq C h^{3-r}\|u\|_{H^{3}}, \quad r \leq 3,
$$

and $\Pi^{*}: S_{h} \rightarrow S_{h}^{*}$ is a piecewise constant interpolation operator.

Then the finite volume element scheme of (1) is defined by: find $u_{h} \in S_{h}$ satisfying

$$
a\left(u_{h}, \Pi^{*} v_{h}\right)=\left(f, \Pi^{*} v_{h}\right), \quad v_{h} \in S_{h} .
$$




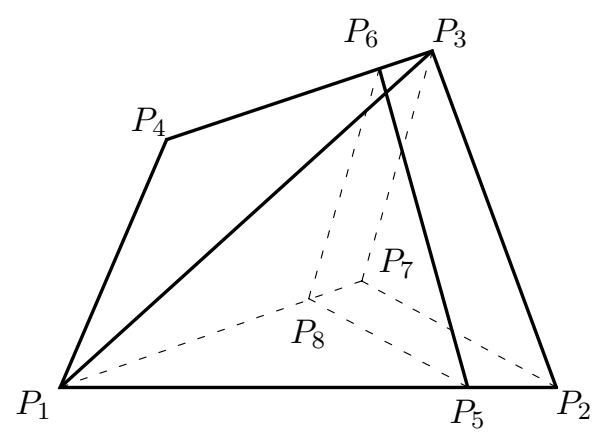

Figure 3. Figure for Lemmas 3.1, 3.2 and 3.4.

\section{Convergence analysis}

In this section, we first investigate several properties of the quadrilateral meshes used in this paper. We will use $P Q$ to denote a line segment, $|P Q|$ to denote its length, and $\overrightarrow{P Q}$ to denote a vector from a point $P$ to a point $Q$.

Lemma 3.1. Suppose that the quadrilateral $P_{1} P_{2} P_{3} P_{4}$ (see Fig. 3) satisfies the almost parallelogram condition (3) and $P_{5} \in\left[P_{1}, P_{2}\right]$ and $P_{6} \in\left[P_{3}, P_{4}\right]$ such that

$$
\frac{\left|P_{2} P_{5}\right|}{\left|P_{2} P_{1}\right|}=\frac{\left|P_{3} P_{6}\right|}{\left|P_{3} P_{4}\right|}=d
$$

where $0 \leq d \leq 1$ is a constant. Then

$$
\left|\overrightarrow{P_{6} P_{5}}+\overrightarrow{P_{1} P_{4}}\right|=O\left(h^{2}\right) .
$$

Proof. In Figure 3, we draw the auxiliary line $P_{3} P_{7}$ parallel to $P_{4} P_{1}$ so that $P_{1} P_{7}$ is parallel to $P_{4} P_{3}$, and draw the auxiliary line $P_{6} P_{8}$ parallel to $P_{3} P_{7}$.

Since in the parallelogram $P_{1} P_{7} P_{3} P_{4}$, the line $P_{6} P_{8}$ is parallel to $P_{3} P_{7}$, then

$$
\frac{\left|P_{7} P_{8}\right|}{\left|P_{7} P_{1}\right|}=\frac{\left|P_{3} P_{6}\right|}{\left|P_{3} P_{4}\right|}=d=\frac{\left|P_{2} P_{5}\right|}{\left|P_{2} P_{1}\right|}
$$

Therefore,

$$
\begin{aligned}
\left|\overrightarrow{P_{6} P_{5}}+\overrightarrow{P_{1} P_{4}}\right| & =\left|\overrightarrow{P_{6} P_{5}}+\overrightarrow{P_{8} P_{6}}\right| \\
& =\left|P_{8} P_{5}\right|=(1-d)\left|P_{7} P_{2}\right|=(1-d)\left|\overrightarrow{P_{7} P_{3}}+\overrightarrow{P_{3} P_{2}}\right| \\
& =(1-d)\left|\overrightarrow{P_{1} P_{4}}+\overrightarrow{P_{3} P_{2}}\right|=(1-d) O\left(h^{2}\right)=O\left(h^{2}\right) .
\end{aligned}
$$

Thus, the proof of Lemma 3.1 is completed.

Lemma 3.2. For any quadrilateral $P_{1} P_{2} P_{3} P_{4}$ (see Fig. 3), let $O_{1}$ and $O_{2}$ denote the midpoints of the two diagonals of $P_{1} P_{2} P_{3} P_{4}$ respectively. Then the condition (3) is equivalent to the following midpoints-distance condition

$$
\left|O_{1} O_{2}\right|=O\left(h^{2}\right)
$$


Proof. If the point $P_{7}$ falls on the line $P_{2} P_{4}$, then

$$
\left|O_{1} O_{2}\right|=\frac{1}{2}\left(\left|P_{4} P_{2}\right|-\left|P_{4} P_{7}\right|\right)=\frac{1}{2}\left|P_{7} P_{2}\right|=\frac{1}{2}\left|\overrightarrow{P_{1} P_{4}}+\overrightarrow{P_{3} P_{2}}\right| .
$$

Otherwise, consider $\triangle P_{2} P_{7} P_{4}$. Since the quadrilateral $P_{1} P_{7} P_{3} P_{4}$ is a parallelogram, then $O_{1}$ and $O_{2}$ are the midpoints of $P_{2} P_{4}$ and $P_{7} P_{4}$. Hence, $O_{1} O_{2}$ is parallel to the line $P_{7} P_{2}$, and

$$
\left|O_{1} O_{2}\right|=\frac{1}{2}\left|P_{7} P_{2}\right|=\frac{1}{2}\left|\overrightarrow{P_{1} P_{4}}+\overrightarrow{P_{3} P_{2}}\right| .
$$

Therefore, if the condition (3) holds, then (13) is true. On the other hand, (13) also implies condition (3).

According to the results in [5], we have the following lemma.

Lemma 3.3. For any quadrilateral $P_{1} P_{2} P_{3} P_{4}$, we denote by $\theta$ the acute angle between the two normals to any two opposite sides. Then the midpoints-distance condition (13) is equivalent to the following small angles condition

$$
\theta=O(h)
$$

Lemma 3.4. Consider the same assumptions as in Lemma 3.2. Then

$$
\left|\angle P_{5} P_{6} P_{4}-\angle P_{4} P_{1} P_{5}\right|=O(h),
$$

and

$$
\angle P_{5} P_{6} P_{4}+\angle P_{6} P_{4} P_{1}=\pi+O(h)
$$

Proof. From Lemma 3.2 and Lemma 3.3, we know that the condition (3) is equivalent to the small angles condition (14). Then, by Lemma 3.1, we know that the quadrilateral $P_{1} P_{5} P_{6} P_{4}$ satisfies the small angles condition (14).

We consider the parallelogram $P_{1} P_{8} P_{6} P_{4}$ to see that

$$
\left|\angle P_{5} P_{6} P_{4}-\angle P_{4} P_{1} P_{5}\right|=\left|\angle P_{5} P_{6} P_{8}-\angle P_{8} P_{1} P_{5}\right| \leq \angle P_{5} P_{6} P_{8}+\angle P_{8} P_{1} P_{5}=\theta_{1}+\theta_{2}=2 O(h)=O(h),
$$

where $\theta_{1}$ is the acute angle between the two normals to the $P_{1} P_{4}$ and $P_{5} P_{6}$, and $\theta_{2}$ is the acute angle between the two normals to opposite sides the $P_{1} P_{5}$ and $P_{4} P_{6}$.

Similarly, we have

$$
\angle P_{5} P_{6} P_{4}+\angle P_{6} P_{4} P_{1}=\pi+\angle P_{5} P_{6} P_{8}=\pi+O(h) .
$$

Thus, the proof is completed.

Now we define a discrete $H^{1}$ semi-norm on $S_{h}$ (see Fig. 2):

$$
\left|u_{h}\right|_{1, h}=\left(\sum_{Q \in T_{h}}\left|u_{h}\right|_{1, h, Q}^{2}\right)^{\frac{1}{2}},
$$

where

$$
\left|u_{h}\right|_{1, h, Q}^{2}=\sum_{i=1}^{4}\left[\left(u_{h}\left(M_{i}\right)-u_{h}\left(P_{i}\right)\right)^{2}+\left(u_{h}\left(P_{i+1}\right)-u_{h}\left(M_{i}\right)\right)^{2}+\left(u_{h}(O)-u_{h}\left(M_{i}\right)\right)^{2}\right] .
$$


Here $P_{i+1}=P_{1}$ if $i=4$. The following lemma gives the equivalence of the normal $H^{1}$ semi-norm and the discrete one.

Lemma 3.5. Assume that the partition $T_{h}$ satisfies the conditions (3) and (4). Then for any $u_{h} \in S_{h}$, there exist positive constants $C_{0}$ and $C_{1}$ independent of $h$ such that

$$
C_{0}\left|u_{h}\right|_{1, h} \leq\left|u_{h}\right|_{H^{1}} \leq C_{1}\left|u_{h}\right|_{1, h}
$$

Proof. According to Lemma 3.2 and $[8,18]$, we have

$$
\begin{aligned}
\left|\hat{u}_{h}\right|_{H^{1}(\widehat{Q})} & \leq C\left\|J_{F_{Q}^{-1}}\right\|_{L^{\infty}(Q)}^{1 / 2}\left|F_{Q}\right|_{W_{\infty}^{1}(\widehat{Q})}\left|u_{h}\right|_{H^{1}(Q)}, \\
\left|u_{h}\right|_{H^{1}(Q)} & \leq C\left\|J_{F_{Q}}\right\|_{L^{\infty}(\widehat{Q})}^{1 / 2}\left|F_{Q}^{-1}\right|_{W_{\infty}^{1}(Q)}\left|\hat{u}_{h}\right|_{H^{1}(\widehat{Q})},
\end{aligned}
$$

where

$$
\begin{array}{r}
\left\|J_{F_{Q}^{-1}}\right\|_{L^{\infty}(Q)} \leq C h_{Q}^{-2}, \quad\left\|J_{F_{Q}}\right\|_{L^{\infty}(\widehat{Q})} \leq C h_{Q}^{2} \\
\left|F_{Q}\right|_{W_{\infty}^{1}(\widehat{Q})} \leq C h_{Q}, \quad\left|F_{Q}^{-1}\right|_{W_{\infty}^{1}(Q)} \leq C h_{Q}^{-1} .
\end{array}
$$

Hence,

$$
C_{0}\left|\hat{u}_{h}\right|_{H^{1}(\widehat{Q})} \leq\left|u_{h}\right|_{H^{1}(Q)} \leq C_{1}\left|\hat{u}_{h}\right|_{H^{1}(\widehat{Q})}
$$

Let $\mathcal{Y}_{u_{h}, Q}$ and $\mathcal{Z}_{u_{h}, Q}$ denote two vectors related to $u_{h}$ on $Q$ satisfying (see Fig. 2)

$$
\begin{aligned}
& \mathcal{Y}_{u_{h}, Q}=\left[u_{h}\left(M_{1}\right)-u_{h}\left(P_{1}\right), u_{h}(O)-u_{h}\left(M_{4}\right), u_{h}\left(M_{3}\right)-u_{h}\left(P_{4}\right),\right. \\
& \left.u_{h}\left(P_{2}\right)-u_{h}\left(M_{1}\right), u_{h}\left(M_{2}\right)-u_{h}(O), u_{h}\left(P_{3}\right)-u_{h}\left(M_{3}\right)\right] \text {, } \\
& \mathcal{Z}_{u_{h}, Q}=\left[u_{h}\left(M_{4}\right)-u_{h}\left(P_{1}\right), u_{h}\left(P_{4}\right)-u_{h}\left(M_{4}\right), u_{h}(O)-u_{h}\left(M_{1}\right),\right. \\
& \left.u_{h}\left(M_{3}\right)-u_{h}(O), u_{h}\left(M_{2}\right)-u_{h}\left(P_{2}\right), u_{h}\left(P_{3}\right)-u_{h}\left(M_{2}\right)\right] \text {. }
\end{aligned}
$$

Let

$$
\mathcal{G}=\frac{1}{30}\left[\begin{array}{ccc}
4 & 2 & -1 \\
2 & 16 & 2 \\
-1 & 2 & 4
\end{array}\right], \quad \mathcal{H}=\frac{1}{3}\left[\begin{array}{cc}
7 & -1 \\
-1 & 7
\end{array}\right]
$$

Here $\mathcal{G}$ is the element mass matrix and $\mathcal{H}$ is produced from the element stiffness matrix of one dimensional quadratic basis. So that by using a computer algebra system (CAS) like maple one can verify the following equality

$$
\left|\hat{u}_{h}\right|_{H^{1}(\widehat{Q})}^{2}=\mathcal{Y}_{u_{h}, Q} \mathcal{H} \otimes \mathcal{G} \mathcal{Y}_{u_{h}, Q}^{T}+\mathcal{Z}_{u_{h}, Q} \mathcal{G} \otimes \mathcal{H Z}_{u_{h}, Q}^{T}
$$

From the properties of tensor product [2,12], maximum and minimum eigenvalues of $H \otimes G$ and $G \otimes H$ can be evaluated by the products of the eigenvalues of the matrices $H$ and $G$ :

$$
\begin{gathered}
\lambda_{\text {max }}(\mathcal{G} \otimes \mathcal{H})=\lambda_{\text {max }}(\mathcal{H} \otimes \mathcal{G})=\frac{38+2 \sqrt{201}}{45}<1.48 \\
\lambda_{\text {min }}(\mathcal{G} \otimes \mathcal{H})=\lambda_{\text {min }}(\mathcal{H} \otimes \mathcal{G})=\frac{19-\sqrt{201}}{30}>0.16
\end{gathered}
$$


So using maximum and minimum eigenvalues and equality

$$
\left|u_{h}\right|_{1, h, Q}^{2}=\mathcal{Y}_{u_{h}, Q} \mathcal{Y}_{u_{h}, Q}^{T}+\mathcal{Z}_{u_{h}, Q} \mathcal{Z}_{u_{h}, Q}^{T},
$$

we obtain the following estimates immediately

$$
\frac{1}{\lambda_{\max }(\mathcal{G} \otimes \mathcal{H})}\left|\hat{u}_{h}\right|_{H^{1}(\widehat{Q})}^{2} \leq\left|u_{h}\right|_{1, h, Q}^{2} \leq \frac{1}{\lambda_{\min }(\mathcal{G} \otimes \mathcal{H})}\left|\hat{u}_{h}\right|_{H^{1}(\widehat{Q})}^{2} .
$$

Combining this estimate with (19) and summing the result over $T_{h}$, we get the desired result.

The following trace theorem [8] will be used in Lemma 3.6 about the continuity of $a\left(\cdot, \Pi^{*} \cdot\right)$ : for any domain $\Omega$ with a Lipschitz boundary, we have

$$
\|u\|_{L^{2}(\partial \Omega)} \leq C\|u\|_{L^{2}(\Omega)}^{\frac{1}{2}}\|u\|_{H^{1}(\Omega)}^{\frac{1}{2}}, \quad \forall u \in H^{1}(\Omega) .
$$

Lemma 3.6. If $u \in \mathrm{H}^{3}(\Omega)$, for any $v_{h} \in S_{h}$, we have

$$
\left|a\left(u-\Pi u, \Pi^{*} v_{h}\right)\right| \leq C h^{2}\|u\|_{H^{3}}\left\|v_{h}\right\|_{H^{1}} .
$$

Proof. In view of (7), we reorder by edges to get

$$
\begin{aligned}
\left|a_{Q}\left(u-\Pi u, \Pi^{*} v_{h}\right)\right|= & \mid \sum_{i=1}^{4}\left(v_{h}\left(M_{i}\right)-v_{h}\left(P_{i}\right)\right) \int_{\partial Q_{M_{i}}^{*} \cap \partial Q_{P_{i}}^{*}} p \nabla(u-\Pi u) \cdot n_{P_{i}} \mathrm{~d} s \\
& +\sum_{i=1}^{4}\left(v_{h}\left(P_{i+1}\right)-v_{h}\left(M_{i}\right)\right) \int_{\partial Q_{P_{i+1}}^{*} \cap \partial Q_{M_{i}}^{*}} p \nabla(u-\Pi u) \cdot n_{M_{i}} \mathrm{~d} s \\
& +\sum_{i=1}^{4}\left(v_{h}(O)-v_{h}\left(M_{i}\right)\right) \int_{\partial Q_{O}^{*} \cap \partial Q_{M_{l}}^{*}} p \nabla(u-\Pi u) \cdot n_{M_{i}} \mathrm{~d} s \mid .
\end{aligned}
$$

It follows from the Cauchy-Schwartz inequality, trace theorem and (10) that

$$
\begin{aligned}
\left|\int_{\partial Q_{M_{i}}^{*} \cap \partial Q_{P_{i}}^{*}} p \nabla(u-\Pi u) \cdot n_{P_{i}} \mathrm{~d} s\right| & \leq C p^{*} h^{1 / 2}\left(\|u-\Pi u\|_{H^{1}\left(\partial Q_{M_{i}}^{*} \cap \partial Q_{P_{i}}^{*}\right)}\right. \\
& \leq C h^{1 / 2}\left(\|u-\Pi u\|_{H^{1}(Q)}^{1 / 2}\|u-\Pi u\|_{H^{2}(Q)}^{1 / 2}\right) \leq C h^{2}\|u\|_{H^{3}(Q)} .
\end{aligned}
$$

According to the definition of the discrete $H^{1}$ semi-norm, it is obvious that

$$
\left|v_{h}\left(M_{i}\right)-v_{h}\left(P_{i}\right)\right| \leq\left|v_{h}\right|_{1, h, Q} .
$$

So we estimate the rest terms similarly and combine the results to obtain

$$
\left|a_{Q}\left(u-\Pi u, \Pi^{*} v_{h}\right)\right| \leq C h^{2}\|u\|_{H^{3}(Q)}\left|v_{h}\right|_{1, h, Q} .
$$

Gathering over all elements and using Lemma 3.5 gives

$$
\left|a\left(u-\Pi u, \Pi^{*} v_{h}\right)\right| \leq C h^{2}\|u\|_{H^{3}}\left|v_{h}\right|_{1, h} \leq C h^{2}\|u\|_{H^{3}}\left\|v_{h}\right\|_{H^{1}} .
$$

Thus, we get the desired result. 
To obtain the coercivity of the bilinear form $a\left(\cdot, \Pi^{*} \cdot\right)$, we will have to use the following two lemmas about partitioned matrix.

Lemma 3.7. Let $\mathcal{A}$ and $\mathcal{B}$ be two $n \times n$ matrices. The constant $\kappa \neq 0$. Then the matrix $\left[\begin{array}{cc}\mathcal{A} & \kappa \mathcal{B} \\ \kappa \mathcal{B}^{T} & \kappa^{2} \mathcal{A}\end{array}\right] \begin{gathered}i s \\ \text { is }\end{gathered}$ positive definite if and only if the matrices $\mathcal{A} \pm \frac{\mathcal{B}+\mathcal{B}^{T}}{2}$ are positive definite.

Proof. First, suppose that the matrix $\left[\begin{array}{cc}\mathcal{A} & \kappa \mathcal{B} \\ \kappa \mathcal{B}^{T} & \kappa^{2} \mathcal{A}\end{array}\right]$ is positive definite. Since $\kappa \neq 0$, for any $\alpha \in R^{1 \times n}$, we note that

$$
(\kappa \alpha, \pm \alpha)\left[\begin{array}{cc}
\mathcal{A} & \kappa \mathcal{B} \\
\kappa \mathcal{B}^{T} & \kappa^{2} \mathcal{A}
\end{array}\right]\left(\begin{array}{c}
\kappa \alpha^{T} \\
\pm \alpha^{T}
\end{array}\right)=2 \kappa^{2} \alpha\left(\mathcal{A} \pm \frac{\mathcal{B}+\mathcal{B}^{T}}{2}\right) \alpha^{T}>0, \forall \alpha \neq 0
$$

So the matrices $\left(\mathcal{A} \pm \frac{\mathcal{B}+\mathcal{B}^{T}}{2}\right)$ are positive definite.

Second, suppose that the matrices $\mathcal{A} \pm \frac{\mathcal{B}+\mathcal{B}^{T}}{2}$ are positive definite. For any $\alpha=\left(\alpha_{1}, \alpha_{2}\right) \in R^{1 \times 2 n}$, where $\alpha_{i} \in R^{1 \times n}$, we have

$$
\begin{aligned}
\alpha\left[\begin{array}{cc}
\mathcal{A} & \kappa \mathcal{B} \\
\kappa \mathcal{B}^{T} & \kappa^{2} \mathcal{A}
\end{array}\right] \alpha^{T}= & \frac{1}{2}\left(\alpha_{1}+\kappa \alpha_{2}\right)\left(\mathcal{A}+\frac{\mathcal{B}+\mathcal{B}^{T}}{2}\right)\left(\alpha_{1}+\kappa \alpha_{2}\right)^{T} \\
& +\frac{1}{2}\left(\alpha_{1}-\kappa \alpha_{2}\right)\left(\mathcal{A}-\frac{\mathcal{B}+\mathcal{B}^{T}}{2}\right)\left(\alpha_{1}-\kappa \alpha_{2}\right)^{T} \\
\geq & 0 .
\end{aligned}
$$

Note that $\left(\alpha_{1}+\kappa \alpha_{2}\right)=\left(\alpha_{1}-\kappa \alpha_{2}\right)=0$ only when $\alpha=0$. So the above inequality becomes an equality only when $\alpha=0$. Therefore, the matrix $\left[\begin{array}{cc}\mathcal{A} & \kappa \mathcal{B} \\ \kappa \mathcal{B}^{T} & \kappa^{2} \mathcal{A}\end{array}\right]$ is positive definite.

As a direct deduction of Lemma 3.7, we have the following result.

Lemma 3.8. Let $\mathcal{A}$ and $\mathcal{B}$ be two partitioned matrices such that $\mathcal{A}=\left[\begin{array}{cc}\mathcal{A}_{1} & \mathcal{A}_{2} \\ \mathcal{A}_{2}^{T} & \mathcal{A}_{1}\end{array}\right]$ and $\mathcal{B}=\left[\begin{array}{cc}\mathcal{B}_{1} & \mathcal{B}_{2} \\ \mathcal{B}_{2} & \mathcal{B}_{1}\end{array}\right]$, where $A_{i}$ and $B_{i}, i=1,2$ are $n \times n$ matrices. The constant $\kappa \neq 0$. Then, the matrix $\left[\begin{array}{cc}\mathcal{A} & \kappa \mathcal{B} \\ \kappa \mathcal{B}^{T} & \kappa^{2} \mathcal{A}\end{array}\right]$ is positive definite if and only if the matrices $\left[\mathcal{A}_{1}+\frac{\mathcal{B}_{1}+\mathcal{B}_{1}^{T}}{2}\right] \pm \frac{1}{2}\left[\left(\mathcal{A}_{2}+\mathcal{A}_{2}\right)^{T}+\left(\mathcal{B}_{2}+\mathcal{B}_{2}^{T}\right)\right]$ and $\left[\mathcal{A}_{1}-\frac{\mathcal{B}_{1}+\mathcal{B}_{1}^{T}}{2}\right] \pm$ $\frac{1}{2}\left[\left(\mathcal{A}_{2}+\mathcal{A}_{2}\right)^{T}-\left(\mathcal{B}_{2}+\mathcal{B}_{2}^{T}\right)\right]$ are positive definite.

Now we make another assumption about the quadrilateral elements in $T_{h}$. Let $\theta_{Q}$ denote any interior angle of $Q \in T_{h}$. Assume that

$$
\left|\cos \theta_{Q}\right| \leq \tau, \quad \forall Q \in T_{h},
$$

where $\tau \geq 0$ is a constant to be determined later.

Lemma 3.9. Suppose that the partition $T_{h}$ satisfies the conditions (2), (3) and (21). Then for sufficiently small $h$, there exists a constant $C_{0}>0$ such that

$$
a\left(u_{h}, \Pi^{*} u_{h}\right) \geq C_{0}\left\|u_{h}\right\|_{H^{1}}^{2}, \quad u_{h} \in S_{h} .
$$


Proof. According to the definition (8), reordering by edges gives

$$
\begin{aligned}
\frac{1}{p(O)} \bar{a}_{Q}\left(u_{h}, \Pi^{*} v_{h}\right)= & \sum_{i=1}^{4}\left(v_{h}\left(M_{i}\right)-v_{h}\left(P_{i}\right)\right) \int_{\partial Q_{M_{i}}^{*} \cap \partial Q_{P_{i}}^{*}} \nabla u_{h} \cdot n_{P_{i}} \mathrm{~d} s \\
& +\sum_{i=1}^{4}\left(v_{h}\left(P_{i+1}\right)-v_{h}\left(M_{i}\right)\right) \int_{\partial Q_{P_{i+1}}^{*} \cap \partial Q_{M_{i}}^{*}} \nabla u_{h} \cdot n_{M_{i}} \mathrm{~d} s \\
& +\sum_{i=1}^{4}\left(v_{h}(O)-v_{h}\left(M_{i}\right)\right) \int_{\partial Q_{Q}^{*} \cap \partial Q_{M_{l}}^{*}} \nabla u_{h} \cdot n_{M_{i}} \mathrm{~d} s .
\end{aligned}
$$

Let $\alpha=\left(\mathcal{Y}_{u_{h}, Q}, \mathcal{Z}_{u_{h}, Q}\right)$ be a composite line vector, where $\mathcal{Y}_{u_{h}, Q}$ and $\mathcal{Z}_{u_{h}, Q}$ are the vectors defined in Lemma 3.5. Denote by

$$
\varphi_{1}(t)=(t-1)(2 t-1), \varphi_{2}(t)=4 t(1-t), \varphi_{3}(t)=t(2 t-1),
$$

the quadratic basis in one dimension. After the the geometry transformation, we integrate the above equation along the reference element edges with respect to tensor product to give that

$$
\frac{1}{p(O)} \bar{a}_{Q}\left(u_{h}, \Pi^{*} u_{h}\right)=\alpha \mathcal{A} \alpha^{T}=\frac{1}{2} \alpha\left(\mathcal{A}+\mathcal{A}^{T}\right) \alpha^{T},
$$

where the partition matrix

$$
\mathcal{A}=\left[\begin{array}{cc}
\mathcal{A}_{1} & \mathcal{A}_{2} \\
\underline{\mathcal{A}_{2}} & \underline{\mathcal{A}_{1}}
\end{array}\right]
$$

Here the matrices $\left(\mathcal{A}_{1}\right)_{6 \times 6}$ and $\left(\underline{\mathcal{A}_{1}}\right)_{6 \times 6}$ reflect the contraction distortion, while the matrices $\left(\mathcal{A}_{2}\right)_{6 \times 6}$ and $\left(\underline{\mathcal{A}_{2}}\right)_{6 \times 6}$ reflect the rotational distortion. The entries of these matrices are specified as follows

$$
\left(\mathcal{A}_{1}\right)_{i j}=\left\{\begin{array}{l}
2 \int_{I_{i}} \frac{\varphi_{j}(\hat{y})}{J_{F_{Q}}\left(\frac{1}{4}, \hat{y}\right)}\left|P_{12} P_{34}\right|^{2}, \quad 1 \leq i, j \leq 3, \\
2 \int_{I_{i-3}} \frac{\varphi_{j-3}(\hat{y})}{J_{F_{Q}}\left(\frac{1}{4}, \hat{y}\right)}\left|P_{21} P_{43}\right|^{2}, \quad 4 \leq i, j \leq 6, \\
0, \quad \text { else }
\end{array}\right.
$$

and

$$
\left(\mathcal{A}_{2}\right)_{i j}=\left\{\begin{array}{l}
C_{j} \int_{I_{i}} \frac{(4 \hat{y}-3)(1-\hat{y})}{J_{F_{Q}}\left(\frac{1}{4}, \hat{y}\right)} \overrightarrow{P_{1} P_{2}} \cdot \overrightarrow{P_{12} P_{43}}+\frac{(4 \hat{y}-3) \hat{y}}{J_{F_{Q}}\left(\frac{1}{4}, \hat{y}\right)} \overrightarrow{P_{4} P_{3}} \cdot \overrightarrow{P_{12} P_{43}}, \quad 1 \leq i, j \leq 3, \\
-C_{j-3} \int_{I_{i}} \frac{(4 \hat{y}-1)(1-\hat{y})}{J_{F_{Q}}\left(\frac{1}{4}, \hat{y}\right)} \overrightarrow{P_{1} P_{2}} \cdot \overrightarrow{P_{12} P_{43}}+\frac{(4 \hat{y}-1) \hat{y}}{J_{F_{Q}}\left(\frac{1}{4}, \hat{y}\right)} \overrightarrow{P_{4} P_{3}} \cdot \overrightarrow{P_{12} P_{43},} \quad 1 \leq i, j-3 \leq 3, \\
-C_{j} \int_{I_{i-3}} \frac{(4 \hat{y}-1)(1-\hat{y})}{J_{F_{Q}}\left(\frac{3}{4}, \hat{y}\right)} \overrightarrow{P_{1} P_{2}} \cdot \overrightarrow{P_{21} P_{34}}+\frac{(4 \hat{y}-1) \hat{y}}{J_{F_{Q}}\left(\frac{3}{4}, \hat{y}\right)} \overrightarrow{P_{4} P_{3}} \cdot \overrightarrow{P_{21} P_{34}}, \quad 1 \leq i-3, j \leq 3, \\
C_{j-3} \int_{I_{i-3}} \frac{(4 \hat{y}-3)(1-\hat{y})}{J_{F_{Q}}\left(\frac{3}{4}, \hat{y}\right)} \overrightarrow{P_{1} P_{2}} \cdot \overrightarrow{P_{21} P_{34}}+\frac{(4 \hat{y}-3) \hat{y}}{J_{F_{Q}}\left(\frac{3}{4}, \hat{y}\right)} \overrightarrow{P_{4} P_{3}} \cdot \overrightarrow{P_{21} P_{34}}, \quad 4 \leq i, j \leq 6, \\
0, \quad \text { else. }
\end{array}\right.
$$


Here $C_{1}=3 / 8, C_{2}=3 / 4$ and $C_{3}=-1 / 8$ and the integral domain $I_{1}=[0,1 / 4], I_{2}=[1 / 4,3 / 4]$ and $I_{3}=[3 / 4,1]$. The matrices $\mathcal{A}_{1}$ and $\mathcal{A}_{2}$ take a similar form as above. In order to study the properties of the matrix $\mathcal{A}$, we shall first introduce an auxiliary matrix $\tilde{\mathcal{A}}$. Since the grids considered in this paper are almost parallelograms, we assume that $Q$ is a parallelogram first, then set $\tilde{\mathcal{A}}=\frac{1}{2}\left(\mathcal{A}+\mathcal{A}^{T}\right)$. Without loss of generality, choose $\theta_{Q}=\angle P_{4} P_{1} P_{2}$ (see Fig. 2). Let $\kappa=\left|P_{1} P_{4}\right| /\left|P_{1} P_{2}\right|$. Therefore,

$$
\tilde{\mathcal{A}}=\frac{\left|P_{1} P_{2}\right|^{2}}{m(Q)}\left[\begin{array}{cc}
\tilde{\mathcal{A}}_{1} & \kappa \frac{\tilde{\mathcal{A}}_{2}+\tilde{\mathcal{A}}_{2}^{T}}{2} \\
\kappa \frac{\tilde{\mathcal{A}}_{2}+\tilde{\mathcal{A}}_{2}^{T}}{2} & \kappa^{2} \tilde{\mathcal{A}}_{1}
\end{array}\right]
$$

with

Here

$$
\tilde{\mathcal{A}}_{1}=\left[\begin{array}{cc}
\tilde{\mathcal{A}}_{11} & 0 \\
0 & \tilde{\mathcal{A}}_{11}
\end{array}\right], \quad \tilde{\mathcal{A}}_{2}=\left[\begin{array}{cc}
\tilde{\mathcal{A}}_{21} & \tilde{\mathcal{A}}_{22} \\
\tilde{\mathcal{A}}_{22} & \tilde{\mathcal{A}}_{21}
\end{array}\right]
$$

$$
\tilde{\mathcal{A}}_{11}=\frac{1}{24}\left[\begin{array}{ccc}
8 & 3 & -1 \\
3 & 22 & 3 \\
-1 & 3 & 8
\end{array}\right], \tilde{\mathcal{A}}_{21}=\frac{\cos \theta_{Q}}{64}\left[\begin{array}{ccc}
-15 & -30 & 5 \\
-12 & -24 & 4 \\
3 & 6 & -1
\end{array}\right], \tilde{\mathcal{A}}_{22}=\frac{\cos \theta_{Q}}{64}\left[\begin{array}{ccc}
3 & 6 & -1 \\
-12 & -24 & 4 \\
-15 & -30 & 5
\end{array}\right]
$$

We can calculate that the minimum principal minor determinant of the matrices $\left(\tilde{\mathcal{A}}_{11}+\frac{\tilde{\mathcal{A}}_{21}+\tilde{\mathcal{A}}_{21}^{T}}{2}\right) \pm$ $\frac{1}{2}\left(\tilde{\mathcal{A}}_{22}+\tilde{\mathcal{A}}_{22}^{T}\right)$ and $\left(\tilde{\mathcal{A}}_{11}-\frac{\tilde{\mathcal{A}}_{21}+\tilde{\mathcal{A}}_{21}^{T}}{2}\right) \pm \frac{1}{2}\left(\tilde{\mathcal{A}}_{22}+\tilde{\mathcal{A}}_{22}^{T}\right)$ is

$$
\frac{17}{192}-\frac{1465}{18432}\left|\cos \left(\theta_{Q}\right)\right|-\frac{3}{64}\left|\cos ^{2}\left(\theta_{Q}\right)\right|-\frac{81}{32768}\left|\cos ^{3}\left(\theta_{Q}\right)\right| .
$$

Let

$$
F(t)=\frac{17}{192}-\frac{1465}{18432} t-\frac{3}{64} t^{2}-\frac{81}{32768} t^{3}
$$

It is easy to verify that the function $F(t)$ is monotone decreasing for $t \in[0,1]$ and there exists a root $t_{0} \in(0,1)$ (this root $\approx 0.7598$ ). So we can choose $\tau<t_{0}$ in $(21)$ such that $F\left(\left|\cos \left(\theta_{Q}\right)\right|\right)>0$. Then the principal minor determinants of the above matrices are all positive. Thus these matrices are positive definite. Now by Lemma 3.8 we know that the matrix

$$
\left[\begin{array}{cc}
\tilde{\mathcal{A}}_{1} & \kappa \frac{\tilde{\mathcal{A}}_{2}+\tilde{\mathcal{A}}_{2}^{T}}{2} \\
\kappa \frac{\tilde{\mathcal{A}}_{2}+\tilde{\mathcal{A}}_{2}^{T}}{2} & \kappa^{2} \tilde{\mathcal{A}}_{1}
\end{array}\right]
$$

is positive definite too with its minimum eigenvalue $\lambda_{\tau}>0$, which is depending on the constant $\tau$. Then using the regularity condition (2), Lemma 3.2 and ([18], Lem. 1), we have

$$
\lambda_{\min }(\tilde{\mathcal{A}}) \geq \lambda_{\tau} \frac{\left|P_{1} P_{2}\right|^{2}}{m(Q)} \geq C \lambda_{\tau}
$$

where $\lambda_{\min }(\tilde{\mathcal{A}})$ denotes the minimum eigenvalue of $\tilde{\mathcal{A}}$. 
We now should consider the difference between the matrix $\tilde{\mathcal{A}}$ on a parallelogram and the matrix $\frac{1}{2}\left(\mathcal{A}+\mathcal{A}^{T}\right)$ on a almost parallelogram. Let $\mathcal{D}=\frac{1}{2}\left(\mathcal{A}+\mathcal{A}^{T}\right)-\tilde{\mathcal{A}}$. Under the condition (3), we see from [13] that

$$
\lim _{h \rightarrow 0} \frac{J_{F_{Q}}(\hat{x}, \hat{y})}{m(Q)}=1
$$

Using Lemma 3.1 and Lemma 3.4, we see that

$$
\left|P_{12} P_{43}\right|=\left|P_{21} P_{34}\right|=\left|P_{1} P_{4}\right|+O\left(h^{2}\right), \quad\left|P_{14} P_{23}\right|=\left|P_{41} P_{32}\right|=\left|P_{1} P_{2}\right|+O\left(h^{2}\right),
$$

and

$$
\begin{aligned}
& \overrightarrow{P_{1} P_{2}} \cdot \overrightarrow{P_{12} P_{43}}=\overrightarrow{P_{4} P_{3}} \cdot \overrightarrow{P_{12} P_{43}}=\overrightarrow{P_{1} P_{2}} \cdot \overrightarrow{P_{21} P_{34}}=\overrightarrow{P_{4} P_{3}} \cdot \overrightarrow{P_{21} P_{34}} \\
& =\overrightarrow{P_{1} P_{4}} \cdot \overrightarrow{P_{14} P_{23}}=\overrightarrow{P_{2} P_{3}} \cdot \overrightarrow{P_{14} P_{43}}=\overrightarrow{P_{1} P_{4}} \cdot \overrightarrow{P_{41} P_{32}}=\overrightarrow{P_{2} P_{3}} \cdot \overrightarrow{P_{41} P_{32}} \\
& =\left|P_{1} P_{2}\right|\left|P_{1} P_{4}\right| \cos \theta_{Q} \cos h+O\left(h^{3}\right) .
\end{aligned}
$$

Thus by (24)-(26), we can verify that when $h$ is small enough,

$$
\left|(\mathcal{D})_{i j}\right| \leq C \frac{(1-\cos h) h^{2}+h^{3}}{m(Q)}, \quad 1 \leq i, j \leq 12 .
$$

Since the partition is regular, then $\frac{h^{2}}{m(Q)} \leq C$. Therefore,

$$
\left|(\mathcal{D})_{i j}\right| \leq C(1-\cos h+h), \quad 1 \leq i, j \leq 12 .
$$

So

$$
\lambda_{\max }(\mathcal{D}) \leq\|\mathcal{D}\|_{\infty} \leq 12 C(1-\cos h+h),
$$

where $\lambda_{\max }(\mathcal{D})$ denotes the maximum eigenvalue of the matrix $\mathcal{D}$.

Combining the results above with the definition (17), we have

$$
\begin{aligned}
\bar{a}_{Q}\left(u_{h}, \Pi^{*} u_{h}\right) & =\frac{p(O)}{2} \alpha\left(\mathcal{A}+\mathcal{A}^{T}\right) \alpha^{T}=p(O) \alpha[\tilde{\mathcal{A}}+\mathcal{D}] \alpha^{T} \\
& \geq p_{*}\left[\lambda_{\min }(\tilde{\mathcal{A}})-\lambda_{\max }(\mathcal{D})\right] \alpha \alpha^{T}=p_{*}\left[\lambda_{\min }(\tilde{\mathcal{A}})-\lambda_{\max }(\mathcal{D})\right]\left|u_{h}\right|_{1, h, Q}^{2} \\
& \geq p_{*}\left[C \lambda_{\tau}-12 C(1-\cos h+h)\right]\left|u_{h}\right|_{1, h, Q}^{2} .
\end{aligned}
$$

From the Lipschitz continuity of $p(X)$, we have

$$
|p(X)-p(O)| \leq L h .
$$

Proceeding similarly as in Lemma 3.6 gives

$$
\left|a_{Q}\left(u_{h}, \Pi^{*} u_{h}\right)-\bar{a}_{Q}\left(u_{h}, \Pi^{*} u_{h}\right)\right| \leq C h\left|u_{h}\right|_{H^{1}(Q)}^{2} .
$$


Hence, using Lemma 3.5 and the Poincaré's inequality, for sufficiently small $h$,

$$
\begin{aligned}
a\left(u_{h}, \Pi^{*} u_{h}\right) & =\sum_{Q \in T_{h}} \bar{a}_{Q}\left(u_{h}, \Pi^{*} u_{h}\right)+\sum_{Q \in T_{h}}\left|a_{Q}\left(u_{h}, \Pi^{*} u_{h}\right)-\bar{a}_{Q}\left(u_{h}, \Pi^{*} u_{h}\right)\right| \\
& \geq p_{*}\left[C \lambda_{\tau}\left(C^{*}\right)^{2}-12 C(1-\cos h+h)\right]\left|u_{h}\right|_{1, h}^{2}-C h\left|u_{h}\right|_{H^{1}}^{2} \\
& \geq C p_{*}\left[C \lambda_{\tau}\left(C^{*}\right)^{2}-12 C(1-\cos h+h)\right]\left|u_{h}\right|_{H^{1}}^{2}-C h\left|u_{h}\right|_{H^{1}}^{2} \\
& \geq C_{0}\left\|u_{h}\right\|_{H^{1}}^{2} .
\end{aligned}
$$

Thus, the proof is completed.

Theorem 3.10. Suppose that $u \in H_{0}^{1}(\Omega) \cap H^{3}(\Omega)$. If the conditions (2), (3) and (21) hold, then for sufficiently small $h$, there exists a positive constant $C$ independent of $h$ such that

$$
\left\|u-u_{h}\right\|_{H^{1}} \leq C h^{2}\|u\|_{H^{3}}
$$

Proof. From (9), (11) and Lemma 3.9, we have

$$
\begin{aligned}
C_{0}\left\|\Pi u-u_{h}\right\|_{H^{1}}^{2} & \leq a\left(\Pi u-u_{h}, \Pi^{*}\left(\Pi u-u_{h}\right)\right) \\
& =a\left(\Pi u-u, \Pi^{*}\left(\Pi u-u_{h}\right)\right) .
\end{aligned}
$$

It follows from Lemma 3.6 that

$$
a\left(\Pi u-u, \Pi^{*}\left(\Pi u-u_{h}\right)\right) \leq C h^{2}\|u\|_{H^{3}}\left\|\Pi u-u_{h}\right\|_{H^{1}} .
$$

Thus,

$$
\left\|\Pi u-u_{h}\right\|_{H^{1}} \leq \frac{C}{C_{0}} h^{2}\|u\|_{H^{3}}
$$

Then from the triangle inequality and interpolation estimates (10), we have

$$
\left\|u-u_{h}\right\|_{H^{1}} \leq\|u-\Pi u\|_{H^{1}}+\left\|\Pi u-u_{h}\right\|_{H^{1}} \leq C h^{2}\|u\|_{H^{3}} .
$$

Thus, the proof of the theorem is completed.

\section{NUMERICAL EXPERIMENT}

In this section, we present some numerical results for the scheme discussed in this paper. Let $\Omega=(0, \pi) \times(0, \pi)$ and $p(X)=(x+1)^{2}+y^{2}$ in problem $(1)$. Let $u(X)=\sin x \sin y$ be the exact solution. The source term $f(X)$ is determined by the above data. Then the performance of the method, in terms of the computed orders of convergence, is reported for two families of meshes (see Fig. 4).

The left one in Figure 4 is a uniform rectangular mesh $T_{h}^{r}$, consisting of a subdivision of the domain into $N \times N$ subsquares for $N=4,8,16, \ldots$ Obviously, $T_{h}^{r}$ satisfies the condition (3).

The right one is a nonuniform quadrilateral mesh $T_{h}^{q}$, consisting of a almost parallelogram grid constructed from a uniform rectangular grid via the mapping

$$
x(i, j)=\frac{\pi i}{N}, \quad y(i, j)=\frac{\pi j}{N}+\frac{\pi}{20} \sin \left(\frac{2 \pi i}{N}\right) \sin \left(\frac{2 \pi i}{N}\right),
$$

where $0 \leq i, j \leq N$. Since the maximum value of the distortion of mesh node positions is proportional to the square of the local mesh size, then for $N=4,8,16, \ldots$, we obtain a $N \times N$ quadrilateral mesh satisfying the almost parallelogram condition (3). 

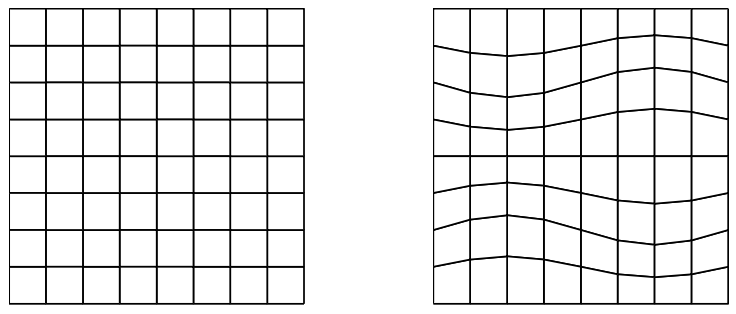

FiguRE 4. Example of the meshes $T_{h}^{r}$ and $T_{h}^{q}$.

TABLE 1. Error estimates and convergence orders on $T_{h}^{r}$.

\begin{tabular}{||c||c|c||c|c||}
\hline$N$ & $e_{1, h}$ & Rate & $e_{2, h}$ & Rate \\
\hline 4 & $3.8605 \times 10^{-2}$ & - & $1.5343 \times 10^{-2}$ & - \\
8 & $9.2577 \times 10^{-3}$ & 2.06 & $3.9196 \times 10^{-3}$ & 1.97 \\
16 & $2.2892 \times 10^{-3}$ & 2.02 & $9.8734 \times 10^{-4}$ & 1.99 \\
32 & $5.7067 \times 10^{-4}$ & 2.00 & $2.4753 \times 10^{-4}$ & 2.00 \\
64 & $1.4261 \times 10^{-4}$ & 2.00 & $6.1921 \times 10^{-5}$ & 2.00 \\
\hline
\end{tabular}

TABLE 2. Error estimates and convergence orders on $T_{h}^{q}$.

\begin{tabular}{||c||c|c||c|c||}
\hline$N$ & $e_{1, h}$ & Rate & $e_{2, h}$ & Rate \\
\hline 4 & $1.2378 \times 10^{-1}$ & - & $2.7408 \times 10^{-2}$ & - \\
8 & $2.7617 \times 10^{-2}$ & 2.16 & $7.3986 \times 10^{-3}$ & 1.89 \\
16 & $6.3542 \times 10^{-3}$ & 2.12 & $1.9442 \times 10^{-3}$ & 1.93 \\
32 & $1.4998 \times 10^{-3}$ & 2.08 & $4.9729 \times 10^{-4}$ & 1.97 \\
64 & $3.5902 \times 10^{-4}$ & 2.06 & $1.2571 \times 10^{-4}$ & 1.98 \\
\hline
\end{tabular}

We compute the error in discrete $H^{1}$ semi-norm and $L^{\infty}$ norm, which is denoted by $e_{1, h}=\left|\Pi u-u_{h}\right|_{1, h}$ and $e_{2, h}=\left\|u-u_{h}\right\|_{L^{\infty}}$ respectively. The numerical order of convergence is then measured by comparing the computed error on two successive mesh level calculations, which is given by the standard formula

$$
\text { Rate }=\log _{2} \frac{e_{i, h}}{e_{i, h / 2}}, \quad i=1,2
$$

Tables 1 and 2 show the results for respectively the case of uniform rectangular mesh $T_{h}^{r}$ and nonuniform quadrilateral mesh $T_{h}^{q}$. We see that the convergence rates in discrete $H^{1}$ semi-norm and $L^{\infty}$ norm for both meshes are second order accurate indeed and the results in these cases display a well agreement with our theoretical results.

Acknowledgements. The author is very grateful to the referees for their careful reading of the manuscript and the constructive comments which improve the paper.

\section{REFERENCES}

[1] R.E. Bank and D.J. Rose, Some error estimates for the box method. SIAM J. Numer. Anal. 24 (1987) 777-787. 
[2] B. Bialecki, M. Ganesh and K. Mustapha, A Petrov-Galerkin method with quadrature for elliptic boundary value problems. IMA J. Numer. Anal. 24 (2004) 157-177.

[3] Z. Cai, On the finite volume element method. Numer. Math. 58 (1991) 713-735.

[4] Z. Cai, J. Mandel and S. McCormick, The finite volume element method for diffusion equations on general triangulations. SIAM J. Numer. Anal. 28 (1991) 392-402.

[5] S.H. Chou and S. He, On the regularity and uniformness conditions on quadrilateral grids. Comput. Methods Appl. Mech. Engrg., 191 (2002) 5149-5158.

[6] S.H. Chou, D.Y. Kwak and K.Y. Kim, Mixed finite volume methods on nonstaggered quadrilateral grids for elliptic problems. Math. Comp. 72 (2002) 525-539.

[7] S.H. Chou, D.Y. Kwak and Q. Li, $L^{p}$ error estimates and superconvergence for covolume or finite volume element methods. Num. Meth. P. D. E. 19 (2003) 463-486.

[8] P.G. Ciarlett, The finite element methods for elliptic problems. North-Holland, Amsterdam, New York, Oxford (1980).

[9] R.E. Ewing, R. Lazarov and Y. Lin, Finite volume element approximations of nonlocal reactive flows in porous media. Num. Meth. P. D. E. 16 (2000) 285-311.

[10] R.E. Ewing, T. Lin and Y. Lin, On the accuracy of the finite volume element method based on piecewise linear polynomials. SIAM J. Numer. Anal. 39 (2001) 1865-1888.

[11] W. Hackbusch, On first and second order box schemes. Computing 41 (1989) 277-296.

[12] R.E. Lynch, J.R. Rice and D.H. Thomas, Direct solution of partitial difference equations by tensor product methods. Numer. Math. 6 (1964) 185-199.

[13] Y. Li and R. Li, Generalized difference methods on arbitrary quadrilateral networks. J. Comput. Math. 17 (1999) 653-672.

[14] R. Li, Z. Chen and W. Wu, Generalized difference methods for differential equations, Numerical analysis of finite volume methods. Marcel Dekker, New York (2000).

[15] F. Liebau, The finite volume element method with quadratic basis functions. Computing 57 (1996) 281-299.

[16] I.D. Mishev, Finite volume element methods for non-definite problems. Numer. Math. 83 (1999) 161-175.

[17] E. Süli, Convergence of finite volume schemes for Poisson's equation on nonuniform meshes. SIAM J. Numer. Anal. 28 (1991) 1419-1430.

[18] E. Süli, The accuracy of cell vertex finite volume methods on quadrilateral meshes. Math. Comp. 59 (1992) 359-382.

[19] M. Tian and Z. Chen, Generalized difference methods for second order elliptic partial differential equations. Numer. Math. J. Chinese Universities 13 (1991) 99-113.

[20] Z.J. Wang, Spectral (finite) volume methods for conservation laws on unstructured grids: basic formulation. J. Comput. Phys. 178 (2002) 210-251.

[21] Z.J. Wang, L. Zhang and Y. Liu, Spectral (finite) volume method for conservation laws on unstructured grids. IV: Extension to two-dimensional systems. J. Comput. Phys. 194 (2004) 716-741.

[22] X. Xiang, Generalized difference methods for second order elliptic equations. Numer. Math. J. Chinese Universities 2 (1983) $114-126$.

[23] M. Yang and Y. Yuan, A multistep finite volume element scheme along characteristics for nonlinear convection diffusion problems. Math. Numer. Sinica 24 (2004) 487-500. 